\title{
New report urges action to eliminate 87,000 cancer cases caused each year by HPV in Europe in women and men
}

$\mathrm{A}$ major new report has called for urgent action to eliminate most of the 87,000 cancer cases caused in papillomavirus) each year across over 50 countries in the WHO European Region.

Viral Protection: Achieving the Possible. A Four Step Plan for Eliminating HPV Cancers in Europe, published by The European Cancer Organisation and produced in collaboration with the professionals of many cancer and other organisations and patients who make up the European Cancer Organisation's HPV Action Network, is the first Europe-wide advocacy initiative to tackle HPV-caused cancers.

Viral Protection highlights that:

$\rightarrow$ A quarter (14 out of 54,26\%) of European region countries still do not vaccinate girls against $\mathrm{HPV}$

$\rightarrow$ Under half ( 26 countries, $48 \%$ ) vaccinate, or are planning to start vaccinating, boys as well as girls

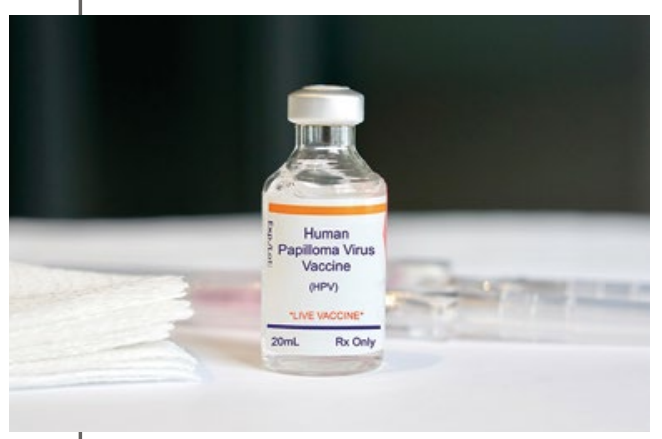

$\rightarrow$ Few countries meet the widely-accepted target of $80 \% \mathrm{HPV}$ vaccination coverage

$\rightarrow 16$ countries have 'opportunistic' cervical cancer screening which means their success depends on the initiative of individual women and their doctors, resulting in lower uptake

$\rightarrow$ Treatment outcomes for cancers caused by HPV vary widely across the region; for women diagnosed with cervical cancer, five-year survival rates range from $80 \%$ in Iceland to 55\% in Poland and Bulgaria

$\rightarrow$ 'Fake news' about HPV vaccination safety, widely shared via social media, has resulted in falls in uptake; in Denmark, it fell from $90 \%$ to $54 \%$.

The report sets out an evidence-based case for action on 28 recommendations on four main fronts:

1. Universal (or 'gender-neutral') HPV vaccination for adolescents should be introduced along with efforts to maximise uptake

2. National organised population-based cervical cancer screening programmes are needed with higher levels of uptake. Screening programmes should use HPV testing technologies which are much more accurate than the traditional Pap smear

3. Cancer treatments must be consistently and equitably offered across all European countries in line with best practice guidelines and with care and support that maximises patients' quality of life

4. Action is needed to improve public and professional awareness and education about HPV in order to improve vaccination and screening uptake and to tackle fake news head-on.

The European Cancer Organisation is urging the European Commission to take action in its new Beating Cancer Plan. The report will also be discussed at the European Cancer Organisation's Summit on 18/19 November where resolutions on HPV will be discussed and voted on and then become part of its continuing policy and advocacy work on this key issue.

Commenting on the call to action, Dr Matti Aapro, President of the European Cancer Organisation, said: 'The new Report, Viral Protection: Achieving the Possible, builds on the World Health Organisation's recently-published global cervical cancer strategy and sets out how elimination of cancers and diseases caused by HPV can be realised through realistic investment and most importantly by building on good practice already in place in many but still too few European countries. This goal, which really is a no-brainer, is achievable through evidencebased steps in four key areas: vaccination, screening, treatment, and public and professional education. We know what to do, so let's seize the moment.'

\section{Free PPE for NHS providers}

BDA members are reminded that PPE is available to NHS providers and orderable every seven days via the PPE portal. To calculate the portal's order limit, practices need to calculate their number of units of dental activity (UDAs) plus number of units of orthodontic activity (UOAs), if any, multiplied by 1.5 per practice per year.

The given example shows that a practice that carries out 2,000 UDAs and 1,000 UOAs per year should report 2,000 plus (1,000 multiplied by 1.5 ) to get a composite metric of 3,500 . The Department of Health has said in its personal protective equipment (PPE) strategy:

'The PPE Portal can be used by all adult social care residential care homes, domiciliary care providers, children's social care settings, GPs, community pharmacies, dentists and optometrists in England. The PPE Portal is intended to meet all COVID-19 needs supplied for free to these NHS contractors, supplementing the PPE supply for business as usual needs that these settings should continue accessing via their normal supply routes. The PPE Portal product range has been expanded and now includes aprons, gloves, type IIR masks, hand hygiene and visors. Additional items, such as respirator masks and gowns will also be made available to eligible service providers via the PPE Portal by October. Weekly order limits are based on provider size, and these will be increased to higher levels using modelled demand of COVID-19 PPE needs.'

Providers should have received an invitation to register sent to your email account registered with Care Quality Commission (CQC), NHS Business Services Authority (BSA), NHS England or the Medicines and Healthcare products Regulatory Agency (MHRA). 